\title{
On a New Method of N-Body Simulations
}

\author{
E. Vilkoviskij \\ Fesenkov Astrophysical Institute, Observatory, Almaty, Kazakhstan \\ Email: vilk@aphi.kz
}

Received July 4, 2012; revised August 15, 2012; accepted August 30, 2012

\begin{abstract}
The theoretical foundation of a new N-body simulation method for the dynamics of large numbers $\left(N>10^{6}\right)$ of gravitating bodies is described. The new approach is founded on the probability description of the physical parameters and a similarity method which permits a manifold reduction of the calculation time for the evolution of "large" systems. This is done by averaging the results of calculations over an ensemble of many "small" systems with total particle number in the ensemble equal to the number of stars in the large system. The method is valid for the approximate calculation of the evolution of large systems, including dissipative systems like AGN containing a super-massive black hole, accretion disc, and the surrounding stellar cluster.
\end{abstract}

Keywords: Galaxies; Nuclei; Methods; N-Body Simulations

\section{Introduction}

At present the methods of modeling systems of many gravitating bodies have been highly refined, but application of these methods to the calculation of very large systems containing $N \sim 10^{6}-10^{11}$ stars (large globular clusters, galaxies and their active nuclei) faces major limitations due to limited computer resources. Furthermore, direct calculation of interactions of every pair of stars demands time proportional to the square of the number of stars (gravitating bodies) $N$ in the system, the whole calculation time (with the calculation time $\Delta t$ for every pair) is proportional to $T \sim \Delta t(N)^{2}$. Usually, one needs to carry the calculations out to a time of the order of the relaxation time $t_{r x}$ of the system, which is proportional to the number of the gravitating bodies $N$. Thus the real "cost" of the calculations behaves as [1]

$$
T\left(N, t_{r x}\right)=\Delta t N^{3}
$$

Such calculations are difficult or impossible for the systems with $N>10^{6}$ due to limited resources of even the most advanced modern supercomputers, with the fast processors with about 1 Petaflops $\left(10^{15} \mathrm{sec}^{-1}\right)$ [1]. Of course, now many approximate methods are developed, such as tree-codes [2], which use the averaged descriptions of the gravity for the distant particle groups. But the classical task of the "direct" calculations of every pair of the gravitating bodies keeps its central role as the most precise method.

In the present paper we discuss a new approximate method for the acceleration of N-body simulations of large stellar systems. We refer to it as the Aldar-Kose method, using the name of a cheery hero of the Kazakh folklore. We discuss its likely areas of application and limitations of the method. In the accompanying paper [3] we show with a sample of simulations, that the method promises essential acceleration of calculations of the evolution models of stellar systems and active galactic nuclei (AGN).

\section{Statistical and Dynamical Equivalence of the Gravitating Systems' Models with Different Initial Particles' Distributions}

Any numerical calculation of N-body dynamics has to be started with definition of the initial coordinates and velocities of all bodies, $\mathrm{X}_{\mathrm{ni}}\left(t_{0}\right), \mathrm{V}_{\mathrm{ni}}\left(t_{0}\right)$, where $i=1,2,3$ corresponds to the Cartesian coordinates and velocity projections of every $\mathrm{n}$-th of the total $\mathrm{N}$ number of the bodies at the starting time $t_{0}=0$. This initial distribution is defined usually with the special procedure of random numbers' generation, which represents some initial quasiequilibrium (with the given full energy of the system) distribution function of the bodies in the common gravity field.

As soon as the initial distributions of the coordinates and velocities of bodies are determined with a random numbers' generation, the condition of the "statistical and dynamical equivalence" of the solutions is implicitly used in any numerical simulations. Let us analyze the concept more distinctly. To avoid non-essential details we simplify the model task supposing equality of the masses of the stars and ignoring direct (contact) stellar collisions, so reducing the task to the "gravitating points" 
dynamics. Also, for simplicity, further we will ignore the problem of "primordial binaries", supposing that their number is determined with the random numbers in the initial conditions.

As the stellar systems are open, the achievement of the exact equilibrium distribution function of the stars' velocities (in Boltzmann's sense) is impossible, because the tendency to statistical equilibrium leads to dissipation of stars with large energies, which determines slow evolution of the system due to the "evaporation" of the stars and the final collapse of the core of the system. The rate of the evolution is determined with the relaxation time of the system [4]

$$
t_{r x}=\frac{0.14 N}{\ln (0.4 N)} t_{c r}
$$

where $N$ is the total number of stars in the system, $\ln (0.4 N)$ is the so-called "Coulomb logarithm" for stellar systems, $t_{c r}$ - the crossing time, determined usually as the time needed for a star to cross the region of the system containing half of the system's mass. Note that the numerical coefficients in (2) are known with the precision $30 \%-50 \%$ only; here we give the most commonly used values.

Let us turn back to the question of equivalence of the evolution processes in the systems with different (in the sense of different realization of random numbers choosing) initial conditions. Of course, the concrete orbits of particles in the systems with different initial conditions will be completely different, but the dynamical behavior and the secular evolution of the distribution functions of the physical parameters of the orbits in both systems will be equivalent in statistical and dynamical sense, if we are interested of distribution functions, but not of the concrete orbits. Note that the quality of modern computers is such that the orbits of the particles, calculated in two simulations with the same initial conditions will be absolutely identical. In contrast, in real stellar systems there are always some random factors, which lead to a mixing (random small deviations) of the orbits. As a result, the distribution functions only, not the concrete stellar orbits may be compared between a model and real systems. Consequently, one may speak not about identity, but only about dynamical and statistical equivalence of a model and real stellar systems. The situation is similar, in a sense, (not literally!) to the quantum-mechanics description: Only the probability description is valid, so the description with a single numerical calculation is "too much deterministic" and, in that sense, non-adequate (fuller analyses of some analogies in quantum mechanics were discussed earlier in [5]).

In fact, the characteristic times of achievement of the "chaotic" (statistical) equilibrium in stellar systems are much shorter than the relaxation time (2), due to the quick dynamical mixture of the systems [6]. As a result, the solutions with different initial conditions in the same system (the "microscopically" different solutions) are supposed to be dynamically and statistically equivalent. As a rule, the problem of different initial condition (in the sense of different random realization) is usually even not discussed, and the solution for the system evolution is realized with a single initial condition of stars' (particles') coordinates and velocities distribution.

With particle numbers more than several thousands, the probability of a deviation from "almost equilibrium" initial distribution is diminishingly small, but the problem becomes actual for the systems with comparatively small number of particles, and for the systems feebly stable relative to the small perturbations. Because of that, the question of equivalence of the numerical solutions became important for comparison of the systems with different numbers of particles, $N_{1}$ and $N_{2}<<N_{1}$.

\section{The Dynamical Equivalence of the Systems with Different Particle Numbers}

Let us consider the question of the dynamical equivalence of the evolution of two systems with different particle numbers, $N_{1}$ and $N_{2}<<N_{1}$. To compare the relaxation processes in the systems it is convenient to use dimensionless unit system (the N-body units, NBU [4]), usually employed in model tasks. In these units, the total mass of the system is $M=1$, its characteristic size $\mathrm{R}=1$, and the gravitation constant $G=1$. In this system, the characteristic particle velocity is $\mathrm{V}=(\mathrm{GM} / \mathrm{R})^{1 / 2}=1$, the characteristic particle crossing time $T_{c r}=\mathrm{R} / \mathrm{V}=1$, and the characteristic time of evolution of the system (the relaxation time) is

$$
T_{r x}=\frac{0.14 N}{\ln (0.4 N)}
$$

We define the concept of the dynamical equivalence of the solutions for the systems with different particle numbers. It is naturally to suppose that the behavior of two systems with different (but large enough) particle numbers $N_{1}$ and $N_{2} \ll N_{1}$ will be dynamically equivalent, if the physical parameters of the systems are compared at equivalent moments of evolution, defined by equal evolution time of systems $T_{e v}=t / T_{r x}$. The evolution times are measured in N-body relaxation time units of both systems (one may call these units the NBEU, the "N-body evolution units" system),

$$
t_{2} / T_{r x 2}=t_{1} / T_{r x 1}=T_{e v}
$$

In the NBU system, the time (and the relaxation times) for both systems are defined through the crossing times, which are equal to unity $\left(T_{c r 1}=T_{c r 2}=1\right)$, and in the NBEU the evolution time is defined through the relaxation time. The relation of the "equivalent dynamical evo- 
lution times" in the NBU system is equal to the relation of their relaxation times:

$$
\frac{t_{2}}{t_{1}}=\frac{T_{r x 2}}{T_{r x 1}}=\frac{N_{2}}{N_{1}} \frac{\ln \left(0.4 N_{1}\right)}{\ln \left(0.4 N_{2}\right)}
$$

In the NBU system, the sizes and dynamical parameters of stellar systems are equal, but the particles in the small $\left(N_{2}\right)$ system have masses $m_{2}=m_{1} N_{1} / N_{2}$, and the volume densities of the particles relate as $N_{1} / N_{2}$. As a result, the crossing times are equal, and the NBU dynamical evolution times in the systems are related as (5). In the NBEU, the time units are relaxation times, so the evolution time of systems in equal (identical) moments of evolution is universal, $T_{e v}=T_{e v 1}=T_{e v 2}$.

The stellar dynamics in star systems is defined with superposition of two processes: The motion along the "smooth" orbits in the averaged gravitation field of the system, and the "quasi-Brownian" motion as the result of random approaches of stars and their density fluctuations. From (4) and (5) it follows that independently of the particle numbers in the systems, their dynamical properties and the time behavior of the physical parameters will be dynamically equivalent in identical moments of the evolutions $T_{e v}$, defined through the relaxation times of both similar systems.

\section{The Scaling Coefficient of the Dynamical Equation for the Dissipative Systems with Different Particle Numbers}

Let us consider the behavior of the stellar systems in the presence of dissipative processes. For definiteness, let it be the active galactic nuclei (AGN), consisting of the compact stellar cluster (CSC) with mass $M$, the central super-massive black hole $(\mathrm{BH})$ with mass $M_{B H}=0.1 M$, and the accretion disc (AD) with the mass $M_{d}=0.01 M$, surrounding the BH. The model is, as they say, the "toy" one - in the sense that the disc parameters (the mass and the rotating moment) are considered to be constants, in spite of the star-disk interactions and the gas and star accretion to the $\mathrm{BH}$; so the total mass and the total rotating moment of the system are not conserved-but it is not important for the further comparative analyses of the $\mathrm{N}$-body tasks (one can imagine that the accretion disk is fed by gas from the outer "obscuring torus" of the AGN). The dynamics of the system, besides the star-star pair interaction, will be defined with gravitation of the black hole and the dissipative star-disk interactions [7]. In the simplest case, the last one is reduced to a friction force, which is directed opposite to the velocity of the star relative to the gas in the disk $\mathbf{V}_{s d}$ and is proportional to the square of the velocity, the square of the stellar radius $R_{s}$ and the gas density $\rho(r)$. So, the dissipative force is

$$
\mathbf{F}=-Q R_{s}^{2} \mathbf{V}_{s d} \mathrm{~V}_{s d} \rho(r)
$$

where $\mathbf{V}_{\mathrm{sd}}=\mathbf{V}_{\mathrm{s}}-\mathbf{V}_{\mathrm{d}}$ is the (vector) difference of the star and gas velocities, $\mathrm{V}_{\mathrm{sd}}$ is the module of the velocity, and $\mathrm{Q}$ is a coefficient of the order of 10 .

In a typical AGN, the mass of the CSC is $M \sim 10^{8} M_{S}$, (of the solar masses), and the number of stars in the system are $N_{1} \sim 10^{8}$. Let us compare the evolution of this "large" (real) system $\left(N_{1}\right.$ stars with masses $\left.M_{S}=M / N_{1}\right)$ to the evolution of a similar "small" (the representing) system with the number of particles $N_{2}=N_{1} / m$ and with the same subsystem's mass relations as in the large one. In $\mathrm{NBU}$, the masses of the particles $M_{2}=m M_{1}$, and the sizes and masses of subsystems are the same as in the large AGN system, with the total masses normalized to 1. The dissipative acceleration per unit mass in the NBU has to be the same in both systems to keep the net velocity change of a particle per disk crossing independent of $\mathrm{m}=N_{1} / N_{2}$ (one can imagine that groups of $\mathrm{m}=N_{1} / N_{2}$ stars are decelerated coherently in the small system). So, the dissipative accelerations of the particles are the same in the both systems,

$$
a=-Q R_{s}^{2} \mathbf{V}_{s d} \mathrm{~V}_{s d} \rho(r) / M_{s}
$$

Now, to compare evolution of the small and large systems, we have to take into account also the difference between the relaxation times in both systems, to keep the dissipative and relaxation time-scales comparable. As in the NBU all sizes and masses of the subsystems (i.e. $\mathrm{CSC}, \mathrm{BH}$ and $\mathrm{AD}$ ), as well as the typical velocities of particles and crossing times are the same in both large and small systems, it is natural to suppose, that behavior (the dynamical evolution) of both systems will be dynamically equivalent, if we compare them at equal moments of evolution (4) of both systems. So, we have to take into account, that the relaxation time in the small system is diminished as compared to the large system according to (5). Due to this, to keep the balance between the effects of the particle-particle and the particle-disk interactions, we must multiply the dissipative acceleration (6) in the small system by the "scaling coefficient" of similarity, $S C=S C\left(N_{2}, N_{1}\right)$, which is equal to the relation of numbers of the disk crossing events by stars per their relaxation times in both systems. So, the scaling coefficient to equalize the dissipative and the relaxation time scales in the small system is

$$
S C\left(N_{2}, N_{1}\right)=\frac{T_{r x 1}}{T_{r x 2}}=\frac{N_{1}}{N_{2}} \frac{\ln \left(0.4 N_{2}\right)}{\ln \left(0.4 N_{1}\right)}
$$

The introduction of the $S C$ to the dissipative force to keep the similarity of evolution of both systems is reasonable at least for the cases when the energy changes of the particles per crossing time (due to the relaxation processes and due to dissipative force per crossing time) are relatively small both in the "large" and the "small" systems. This circumstance can limit the relation $\mathrm{m}=N_{1} / N_{2}$ 
of the particle numbers in the real and the "representing" model systems.

Now let us take into account that the particle numbers $N_{2}$ and $N_{1}$ in both systems can change in time both because of the accretion of the stars to the central $\mathrm{BH}$ (the intensity of the process is essentially increased in AGN as the result of star-disk interactions [7]) and due to the "evaporation" of the stars from the systems. These effects can be taken into account with introduction of the variable relaxation and evolution times. Denoting $i=1,2$ for the large and the small systems, and using $t$ for the dynamical time, we can write:

$$
\begin{aligned}
& T_{r x, i}(t)=\frac{0.14 N_{i}(t)}{\ln \left(0.4 N_{i}(t)\right)} \\
& T_{e v}(t)=\int_{0}^{t} \frac{\ln \left(0.4 N_{i}\left(t^{\prime}\right)\right)}{0.14 N_{i}\left(t^{\prime}\right)} \mathrm{d} t^{\prime}
\end{aligned}
$$

where $N_{i}\left(t^{\prime}\right)=N_{i}-N_{b h, i}\left(t^{\prime}\right)-N_{\text {out }, i}\left(t^{\prime}\right) ; N_{b h, i}\left(t^{\prime}\right)$ and $N_{\text {out }, i}\left(t^{\prime}\right)$ are the numbers of stars captured to the $\mathrm{BH}$ and evaporated from the systems. Remind that $T_{e v}(t)$ are equal in both systems by definition.

The time-dependent $N_{1}(t)$ and $N_{2}(t)$ have to be introduced to the $S C$ as well:

$$
\begin{aligned}
S C\left(N_{2}(t), N_{1}(t)\right) & =\frac{T_{r x 1}(t)}{T_{r x 2}(t)} \\
& =\frac{N_{1}(t)}{N_{2}(t)} \frac{\ln \left(0.4 N_{2}(t)\right)}{\ln \left(0.4 N_{1}(t)\right)}
\end{aligned}
$$

Here the number $N_{1}(t)$ is present, which is supposed to be unknown in the A-K model simulation and has to be defined through $N_{2}(t)$. For that, let us transform (10) from the NBU time units to the evolution time units NBEU, defined with (9). Then we have

$$
\begin{aligned}
S C\left(N_{2}, N_{1}, T_{e v}\right) & =\frac{T_{r x 1}\left(T_{e v}\right)}{T_{r x 2}\left(T_{e v}\right)} \\
& =\frac{N_{1}\left(T_{e v}\right)}{N_{2}\left(T_{e v}\right)} \frac{\ln \left(0.4 N_{2}\left(T_{e v}\right)\right)}{\ln \left(0.4 N_{1}\left(T_{e v}\right)\right)}
\end{aligned}
$$

As, by definition,

$N_{1}\left(T_{e v}\right) / N_{1}\left(T_{e v}=0\right)=N_{2}\left(T_{e v}\right) / N_{2}\left(T_{e v}=0\right)$, finally we have

$$
S C\left(N_{2}, N_{1}, T_{e v}\right)=\frac{N_{1}}{N_{2}} \frac{\ln \left(0.4 N_{2}\left(T_{e v}\right)\right)}{\ln \left(0.4\left(N_{1} / N_{2}\right) N_{2}\left(T_{e v}\right)\right)}
$$

where $N_{1} / N_{2}$ is the relation of initial particle numbers in the systems. So, in the approximation, the $S C$ depends on $t$ very slowly (logarithmically).

Using (12), one can calculate the evolution of the small system (which "represents" the evolution of the large system), using only the initial relation of the parti- cle numbers in both systems, and the universal $T_{e v}$, equal in both systems.

Here we can see the meaning of approximating representation of the large system by the small one: The small system "represents" the large one, but not literally. The main difference is that we change many disk-crossing events of small particle in the large system, to one crossing event (with the $S C$ ) of a large particle in the small system. In the first case the crossing point can move along the disk radius (due to scattering of the orbits and small distortion of the spherical symmetry of the $\mathrm{BH}$ potential by the disk gravity), slowly changing the conditions of the crossings. In the "small" system the process is changed with one event at the single crossing point. This difference is the main reason limiting the relation $\mathrm{m}=N_{1} / N_{2}$ (see discussion below in the Part 6 of the paper).

So, the dynamical equivalence of the small and the large dissipative systems is approximate only and has to be carefully controlled. The corresponding scaling coefficients can be obtained for other processes as well, like the direct (contact) stellar collisions, stellar evolution and other processes.

\section{The Statistical Equivalence of the Solutions for the Systems with Different Numbers of Particles: The Aldar-Kose Method}

It is obvious that the above-described dynamical equivalence of the small and the large systems does not mean the statistical equivalence of the model solutions for them, because the statistical precisions of the solutions are related as $\left(N_{2} / N_{1}\right)^{1 / 2}$. The natural way to increase the statistical precision of solutions for the small systems is the manifold repeated calculations among the ensemble of small systems. Providing the simulations of small systems $\mathrm{m}=\left(N_{1} / N_{2}\right)$ times with different (randomly defined) initial conditions, and averaging the results over the full ensemble of the solutions at identical $T_{e v}$ moments, one will obtain the demanded increasing of the precision to the statistical precision, achieved with a single model solution of one large system.

So, we conclude that the mean values of physical parameters, obtained with averaging over $\mathrm{m}=N_{1} / N_{2}$ model solutions for the ensemble of dynamically equivalent "small" systems (every with $N_{2}$ particles), are equivalent to those obtained with one solution for the "large" system ( $N_{1}$ particles), both in the dynamical and statistical senses.

This conclusion has principal meaning for substantiation of the proposed new method (the A-K method) for the model simulations of evolution of the stellar systems and AGN, containing compact stellar clusters. Remind that the "classical" direct method of the N-body simulations of the systems evolution demands spending of the 
calculation time proportional to the third power of the particle number in the system (1). With the A-K-method of averaging over ensemble of $\mathrm{m}=N_{1} / N_{2}$ subsystems, the total demanded time is

$$
T_{A K}\left(N_{1}\right) \sim \Delta t\left(N_{1} / N_{2}\right)\left(N_{2}\right)^{3}=\Delta t N_{1} N_{2}^{2},
$$

where $N_{1}$ and $N_{2}$ are the particle numbers in the "large" and the "small" systems.

The relation of the calculation times demanded in the A-K method and the "direct" (Usual Calculations) method is

$$
T_{A K}\left(N_{1}\right) / T_{U C}\left(N_{1}\right)=\left(\Delta t_{A K} / \Delta t_{U C}\right)\left(N_{2} / N_{1}\right)^{2}
$$

where $\left(\Delta t_{A K} / \Delta t_{U C}\right)$ is the relation of calculation times of the both methods, demanded for equal time intervals in the NBU.

\section{Possible Limitations of the A-K Method}

Let us discuss possible limitations of the method. Estimations of the calculation times demanded for equal dynamical times $\left(\Delta t_{A K} / \Delta t_{U C}\right)$ in both $A K$ and $U C$ methods depend on many factors, including the precision demanded in the task. The point is that in the "small" system, imitating (representing) the behavior of the "large" system, the gravitation accelerations of close particles are larger than those in the large system. Besides, as mentioned in the Part 5, the limitations for the $S C\left(N_{2}, N_{1}\right)$ do exist. These circumstances can demand diminishing of the integration steps and increasing of the calculation time in the A-K method. But these problems appear in the limited areas of particles close encounters and the disk crossing in the large gas density areas. The encounter problem can be avoided with the "softening parameter" $\epsilon$ in the potential $U \sim 1 /(r+\epsilon)$, and one can control the gas density in the disk as well. And, last but not least, because of poor knowledge of the accretion disks' structure, one usually needs not too much precision of the star-disk interaction calculations close to the $\mathrm{BH}$. All these circumstances increase possible using of the AK-method for the approximating calculation for the sake of finding the quality differences of the "evolution tracks" of AGN with different initial properties.

The more definite conclusion about influences of all such factors can be obtained only with corresponding numerical experiments. The model calculations performed by us [3] had partly dispelled our anxieties regarding the possible slowing down of the calculations of the "small" systems evolution. The possibilities of our computer are limited, so the largest systems we can calculate (for several days) up to the evolution time $T_{e v} \sim 2$ with the direct (UC) method are those with $N_{1} \leq 32 \times 10^{3}$ particles.

Here, just for illustration, we show a result of calculation of the stellar orbits' inclinations to the plane of the accretion disk $(\cos (\mathrm{i}))$ for the inner region of the AGN with size equal to the outer disk radius $\left(\mathrm{R}_{\mathrm{ad}}=0.22\right)$, at the moment of evolution time $T_{e v}=1.7$ (Figure 1). The result for the direct simulation with $N=16 \times 10^{3}$ (16 K particles) is shown with red line, and the A-K calculations with numbers of the representing ensemble members $m=4$ and $\mathrm{m}=8$ are shown with green and blue colors (accordingly, for $N_{2}=4 \mathrm{~K}$ and $2 \mathrm{~K}$ ). The "truncated" A-K variant (averaged over 4 instead of 8 solutions with $\mathrm{m}=8$ ) is shown with agenda broken line. One can see that the distributions are indistinguishable, with larger noise in the last case.

It appears that the direct calculations of "large" systems with $N_{1}=16 \times 10^{3}$ (and $N_{1}=32 \times 10^{3}$, see [3]) particles, and with the particle numbers in the "small" subsystem $N_{2} \geq(2-4) \times 10^{3}$, both (A-K and $U C$ ) methods lead to practically equal results, but with smaller representing systems $N_{2}\left(N_{2}<2 \times 10^{3}\right)$ the inaccuracy and duration of the A-K calculations are quickly increased. More detailed numerical investigations of the A-K applications are presented and discussed in the paper [3].

\section{Conclusions}

The main conclusions are:

1) The proposed new A-K similarity method for modeling large stellar systems and AGN evolution promises an essential improvement in the calculation time as compared to the direct N-body simulation. The gain increases with the number $m$ of small systems in the ensemble. Though the maximal $m$ is limited by the condition on the dynamical equivalence precision, the A-K method can essentially increase the possibilities of model calculations with "medium" power (and price) computers;

2) The proposed method is approximate, but it is enough for the quality investigations of evolution of the complicated systems like AGN, where the main interest

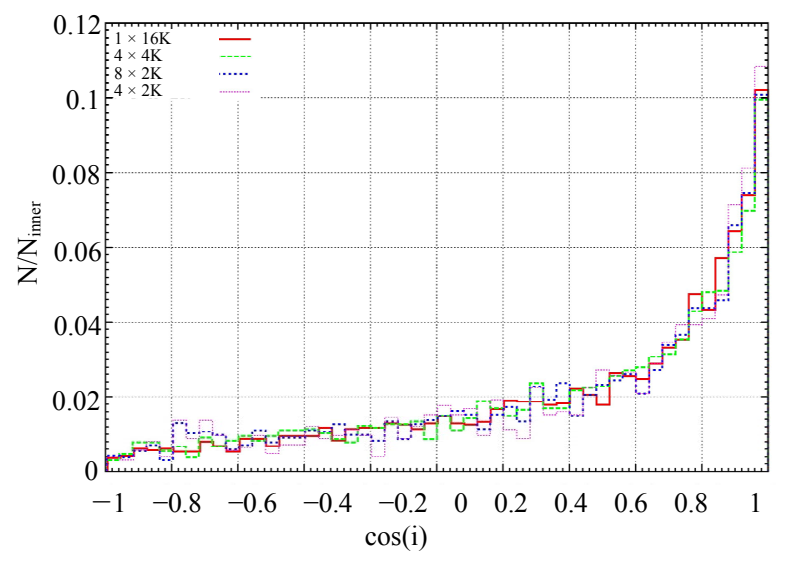

Figure 1. The distribution of the $\cos (i)$ of the stellar orbits' declinations at the evolution time $T_{e v}=1.7$. 
is the qualitative differences between evolution tracks of the systems with different initial parameters;

3 ) Since the statistical precision of solutions for different physical parameters behaves as $\sigma \sim 1 /\left(N_{1}\right)^{1 / 2}$, the achievable formal precision of the A-K method is usually far greater than those obtained from observations. This permits further optimization of the method using "truncated" A-K method: The number of the small systems for averaging the physical parameters in the ensemble can be chosen to be much smaller than $m=N_{1} / N_{2}$, which further reduces the demands on calculation time compared to the "complete" (full) A-K method.

4) Numerical experiments with more powerful computers are necessary to investigate the exact possibilities and limits of applications of the method for the solutions of specific problems of stellar dynamics and AGN evolution.

In conclusion, it should be noted that our formulae (8 12) are approximate, as we use the concept of the "total" relaxation time, which possibly is only approximate when a stellar system changes its spatial structure and symmetry. This issue will be investigated in future works.

\section{Acknowledgements}

The author is grateful to M. A. Makukov for the numerical calculations (to be published in the accompanying paper [3]), to I. S. Vilkoviskij for useful discussion of the variable $T_{e v}(t)$, and to all participants of the "STAR-
DISK" project (P. Berczik, R. Spurzem, Ch. Omarov, M. Makukov, D. Yurin, A. Just) for the elaboration of the computer code for the direct N-body simulations of AGN evolution with a single representing stellar system $(m=1)$.

\section{REFERENCES}

[1] P. Hut, "Dense Stellar Systems as Laboratories for Fundamental Physics," New Astronomy Reviews, Vol. 54, No. 3-6, 2010, pp. 163-172. doi:10.1016/j.newar.2010.09.009

[2] S. Aarseth, "From NBODY1 to NBODY6: The Growth of an Industry," The Publications of the Astronomical Society of the Pacific, Vol. 111, No. 765, 1999, pp. 13331346. doi: $10.1086 / 316455$

[3] E. Vilkoviskij and M. Makukov, International Journal of Astronomy and Astrophysics, 2012.

[4] D. Heggie and P. Hut, "The Gravitational Million-Body Problem," Cambridge University Press, Cambridge, 2003. doi:10.1017/CBO9781139164535

[5] W. Saslaw, "Gravitational Physics of Stellar and Galactic Systems," Cambridge University Press, Cambridge, 1987.

[6] D. Lynden-Bell, "Statistical Mechanics of Violent Relaxations in Stellar Systems," Monthly Notices of the Royal Astronomical Society, Vol. 136, 1967, pp. 101-121.

[7] E. Vilkoviskij and B. Czerny, "The Role of the Central Stellar Cluster in AGN," Astronomy and Astrophysics, Vol. 387, 2002, pp. 804-819. doi:10.1051/0004-6361:20020255 